\title{
TOA Estimation in UWB: Comparison between Time and Frequency Domain Processing
}

\author{
Eva Lagunas ${ }^{1}$, Lorenzo Taponecco $^{3}$, Montse Nájar $^{1,2}$, and Antonio D'Amico ${ }^{3}$ \\ 1 Department of Signal Theory and Communications, Universitat Politècnica de \\ Catalunya (UPC), C/ Jordi Girona 1-3, 08034, Barcelona, SPAIN, \\ \{eva. lagunas, montse.najar\}@upc.edu \\ 2 Centre Tecnològic de Telecomunicacions de Catalunya (CTTC), Av. Canal Olmpic, \\ Castelldefels, Spain \\ 3 Department of Information Engineering, Università di Pisa, Via G. Caruso 16, \\ 56122, Pisa , ITALY, \\ \{lorenzo.taponecco, antonio.damico\}@iet.unipi.it
}

\begin{abstract}
A comparison between time and frequency domain TOA estimators compliant with the 802.15.4a Standard has been made. The time domain estimator is done in two stages. One has the goal of identify the first pulse in each symbol interval and the other aims to acquire the start of frame delimiter. The frequency domain estimator is also based in two stages. A simple coarse estimation stage provides the symbol synchronization required by the fine estimation stage which finds the first delay of the first arriving path in a power delay profile developed in the frequency domain by using high resolution spectral estimation techniques. An accuracy of few centimeters is asymptotically achieved by both estimators. While time domain estimator shows slightly more accurate results at high values of SNR, frequency domain estimators works slightly better at low SNR. ${ }^{1}$
\end{abstract}

Key words: Time of Arrival estimation, Ultra-Wide Band, IEEE 802.15.4a Standard, frequency domain, time domain

\section{Introduction}

Localization and Positioning represents one of the main themes that are being developed in the field of wireless communications. One of the existing technologies that offers better features related to the location applications is Ultra-Wideband (UWB). UWB signals can be used for accurate time of arrival (TOA) based ranging because of their high time resolution. The TOA approach estimates the time of flight of the first arriving path and thus acquires the distance information

\footnotetext{
${ }^{1}$ This work was partially supported by the Departament d'Universitats, Recerca i Societat de la Informació de la Generalitat de Catalunya, by the Catalan Government under grant 2009 SGR 891, by the Spanish Government under project TEC200806327-C03 (MULTI-ADAPTIVE) and by the European Commission under project NEWCOM++ (ICT-FP7-216715)
} 
between a pair of nodes by multiplying the travel time by the speed of the light. The TOA estimation accuracy depends on how precisely the receiver can discriminate the first arriving signal, which in a multipath environment may not be the strongest.

This work discusses two ranging algorithms for impulse radio UWB signals compliant with the IEEE 802.15.4a standard. The first one is described in [1]- [2]. It proposes a ranging algorithm for non-coherent receivers based on the preamble of the IEEE 802.15.4a standard. The ranging problem consists in estimating the TOA of the first pulse of the PHR (the ranging marker). The estimation is done in a two-step procedure which first leads to a coarse estimate and then to a finer results. The second estimator considers a frequency domain approach based on the algorithm proposed in [3]- [4] which has been adapted to comply with the IEEE 802.15.4a. The estimator is performed in two stages. First a coarse estimation is done in order to provide the time reference for symbol synchronization and then, working in the frequency domain, a high resolution estimation of the first arriving path is done in the second stage.

\section{System Model}

The standard specifies two optional signalling formats based on Impulse-Radio UWB (IR-UWB) and Chip Spread Spectrum (CSS). The IR-UWB option targets mainly for ranging whereas the CSS signals have better features for data communication. Since we investigate ranging for the IEEE 802.15.4a standard we only focus on the IR-UWB option. The frame format structure is shown in Fig. 1.

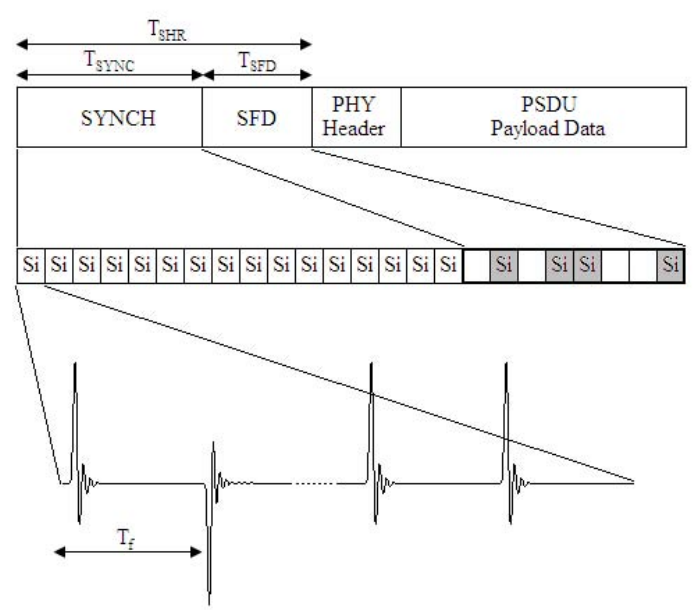

Fig. 1. Frame format structure 
The standard packet consists of a synchronization header (SHR) preamble, a physical layer header (PHR) and a data field (PSDU). The SHR preamble is composed of the ranging (SYNCH) preamble and the start of frame delimiter (SFD). The ranging preamble can consist of 16,64,1024,4096 symbols. The underlying symbol of the ranging preamble uses one of the length-31 ternary sequences, $c_{i}$. Each $c_{i}$ of length $K_{p b s}=31$ contains 15 zeros and 16 non-zero codes, and has the desired property of perfect periodic autocorrelation. The PHR consists of 19 bits and conveys information necessary for a successful decoding of the packet to the receiver.

The mathematical model of the signal transmitted during the SHR is,

$$
s(t)=\sum_{k=0}^{N_{S Y N C}+N_{S F D}-1} a_{k} \psi\left(t-k T_{\text {sym }}\right)
$$

where $N_{S Y N C}$ and $N_{S F D}$ are the lengths of the SYNC and the SFD and $T_{\text {sym }}$ is the symbol duration. Coefficients $a_{k}$ are all equal to 1 during the SYNC while they take values $\{-1,0,+1\}$ during the SFD. Finally $\psi(t)$ is expressed as,

$$
\psi(t)=\sum_{j=0}^{K_{p b s}-1} c_{j} p\left(t-j T_{f}\right)
$$

In this equation $p(t)$ is an ultrashort pulse (monocycle) and $T_{f}=T_{s y m} / K_{p b s}$ is the pulse repetition period.

Signal $s(t)$ propagates through an L-path fading channel whose response to $p(t)$ is $\sum_{l=0}^{L-1} h_{l} p\left(t-\tau_{l}\right)$. Note that it is assumed that the received pulse from each l-th path exhibits the same waveform but experiences a different fading coefficient, $h_{l}$, and a different delay, $\tau_{l}$. Without loss of generality we assume $\tau_{0} \leq \tau_{1} \leq \ldots \leq \tau_{L-1}$. The received waveform can be written as,

$$
r(t)=\sum_{k=0}^{N_{S H R}-1} \sum_{j=0}^{K_{p b s}-1} \sum_{l=0}^{L-1} a_{k} c_{j} h_{l} p\left(t-T_{k}^{j}-\tau_{l}\right)+w(t)
$$

where $w(t)$ is thermal noise with two-sided power spectral density $N o / 2, T_{k}^{j}=$ $j T_{f}+k T_{\text {sym }}$ and $N_{S H R}=N_{S Y N C}+N_{S F D}$.

\section{Frequency Domain TOA Estimation}

Next, the frequency domain TOA estimator [3] is described in detail for completeness. The block diagram of the frequency domain TOA estimator is sketched in Fig. 2. For the purpose of describing the estimation algorithm the receiver assumes an ideal Band Pass Filter (BPF) sampled at Nyquist rate, followed by a Discrete Fourier Transform (DFT) module that transforms the signal to the frequency domain. The frequency samples are processed in the TOA estimator stage without knowledge of the specific pulse shape at the output of the BPF. 


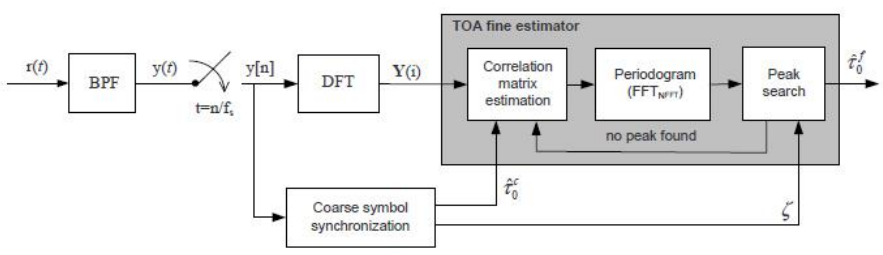

Fig. 2. Block diagram of the non-coherent receiver

The fine TOA estimator requires a previous stage that provides a coarse symbol synchronization. Note that this receiver scheme is a non-coherent receiver.

The signal associated to the $\mathrm{j}$-th transmitted pulse corresponding to the $\mathrm{k}$-th symbol, in the frequency domain yields:

$$
Y_{j}^{k}(w)=\sum_{l=0}^{L-1} a_{k} c_{j} h_{l} S_{j}^{k}(w) e^{-j w \tau_{l}}+V_{j}^{k}(w)
$$

with,

$$
S_{j}^{k}(w)=\tilde{P}(w) e^{-j w\left(\left(k N_{f}+j\right) T_{f}\right.}
$$

where $\tilde{P}$ denotes the Fourier Transform of $\tilde{p}(t)$ which is the received pulse waveform at the output of the $\mathrm{BPF}$ and $N_{f}$ is the number of frames per symbol. $V_{j}^{k}(w)$ is the noise in the frequency domain associated to the $\mathrm{j}$-th frame interval correspondig to the k-th symbol. Sampling (4) at $w_{n}=w_{0} n$ for $n=0,1, \ldots, N-1$ and $w_{0}=2 \pi / N$ and rearranging the frequency domain samples $Y_{j}^{k}[n]$ into the vector $\mathbf{Y}_{j}^{k} \in \mathbb{C}^{N \times 1}$ yields,

$$
\mathbf{Y}_{j}^{k}=a_{k} c_{j} \mathbf{S}_{j}^{k} \mathbf{E}_{\tau} \mathbf{h}+\mathbf{V}_{j}^{k}
$$

where the matrix $\mathbf{S}_{j}^{k} \in \mathbb{C}^{N \times N}$ is a diagonal matrix whose components are the frequency samples of $S_{j}^{k}(w)$ and the matrix $\mathbf{E}_{\tau} \in \mathbb{C}^{N \times L}$ contains the delaysignature vectors associated to each arriving delayed signal,

$$
\mathbf{E}_{\tau}=\left[\mathbf{e}_{\tau_{0}} \ldots \mathbf{e}_{\tau_{j}} \ldots \mathbf{e}_{\tau_{L-1}}\right]
$$

with $\mathbf{e}_{\tau_{j}}=\left[1 e^{-j w_{0} \tau_{j}} \ldots e^{-j w_{0}(N-1) \tau_{j}}\right]^{T}$. The channel fading coefficients are arranged in the vector $\mathbf{h}=\left[h_{0} \ldots h_{L-1}\right]^{T} \in \mathbb{C}^{L \times 1}$, and the noise samples in vector $\mathbf{V}_{j}^{k} \in \mathbb{C}^{N \times 1}$.

Estimation of the TOA from the noisy observation $y(t)$ without knowledge of the specific pulse shape $\tilde{p}(t)$ is the goal of the algorithm [4]- [3]. First, a simple coarse estimation stage that provides the time reference for a symbol synchronization and estimates the threshold used in the TOA algorithm is developed. The frequency domain samples are then processed in the fine TOA estimator stage. The resolution of the algorithm is given by the pulse time width. 


\subsection{Coarse TOA Estimation}

The coarse estimation consists of an energy estimator and a simple search algorithm that identifies the beginning of the symbol by applying a minimum distance criterion. Since the signal structure in the Standard does not include a time hopping sequence, the minimum distance criterion is applied in this case based on the ternary sequence knowledge, $c_{i}$, at symbol level.

It is assumed that the acquisition begins at any point of the SYNCH preamble $t_{0}$ and lasts $K_{s}+1$ symbols. Note that the acquisition window duration is defined one symbol longer than the number of symbols considered for the fine timing estimation.

To find the beginning of the next symbol the frame number which the first detected pulse belongs to is needed. Lets denote $y[m]=y\left(m T_{s}\right)$ the discrete-time received signal, where $T_{s}$ is the sampling period. The time domain samples of the received signal in the i-th frame time interval are defined as,

$$
y_{\text {frame }, i}[n]=y\left[(i-1) K_{f}+n\right] \text { for } \quad n=1, \ldots, K_{f}
$$

where $i=1, \ldots, N_{f}\left(K_{s}+1\right)$. Rearranging the time domain samples $y_{\text {frame }, i}[n]$ in the vector $\mathbf{y}_{\text {frame }, i} \in \mathbb{C}^{K_{f} \times 1}$, being $K_{f}=\left\lfloor T_{f} / T_{s}\right\rfloor$ the number of samples in a frame interval, the energy at each frame interval in one symbol period is obtained averaging for each of the $N_{f}$ frames, over all $K_{s}+1$ symbols in the acquisition interval. That is,

$$
E_{\text {frame }, j}=\sum_{k=0}^{K_{s}}\left\|\mathbf{y}_{\text {frame }, j+31 k}\right\|^{2} \quad j=1, \ldots, N_{f}
$$

Although the energy estimation is done in the temporal domain, it can be also obtained in the frequency domain. Then the algorithm searches the 16 maxima corresponding to the 16 frames containing pulses and estimates the ternary sequence $\hat{c}_{i}$. From the original ternary sequence $c_{i}$ it is defined the vector $\mathbf{d}$ as a vector composed of the 16 positions of the sequence $c_{i}$ containing \pm 1 . Then it is defined circulant matrix $\Delta_{\rho_{c_{i}}}$ whose rows contain the relative delays in number of frame intervals between two consecutive pulses within a symbol period. Each row is a shifted version of the previous one. More specifically, defining $\rho_{c_{i}}(n)=d(n+1)-d(n)-1$ for $n=1, \ldots, 15$ and $\rho_{c_{i}}(16)=31-d(16)+d(1)$ as the number of frames between two consecutive transmitted pulses, the circulant matrix $\Delta_{\rho_{c_{i}}}$ is given by,

$$
\Delta_{\rho_{c_{i}}}=\left[\begin{array}{ccccc}
\rho_{c_{i}}(1) & \rho_{c_{i}}(2) & \ldots & \rho_{c_{i}}(15) & \rho_{c_{i}}(16) \\
\rho_{c_{i}}(2) & \rho_{c_{i}}(3) & \ldots & \rho_{c_{i}}(16) & \rho_{c_{i}}(1) \\
\vdots & \vdots & \ddots & \vdots & \vdots \\
\rho_{c_{i}}(16) & \rho_{c_{i}}(1) & \ldots & \rho_{c_{i}}(14) & \rho_{c_{i}}(15)
\end{array}\right]
$$

Hence, with the estimated ternary sequence $\hat{c}_{i}$ it is conformed the vector $\hat{d}$ which contains the estimated pulses positions or, in other words, which contains the 
16 positions of the estimated sequence $\hat{c}_{i}$ containing \pm 1 . Therefore, the relative distance between the 16 estimated positions of the pulses form the vector,

$$
\Delta d=\left[\hat{\rho}_{c_{i}}(1) \hat{\rho}_{c_{i}}(2) \ldots \hat{\rho}_{c_{i}}(15) \hat{\rho}_{c_{i}}(16)\right]
$$

If we denote the first pulse within the symbol with the number 1 , the second with the number 2 and so on until the sixteenth pulse with the number 16 , then the estimated number of the first detected pulse $\eta \in\{1, \ldots, 16\}$ is carried out by finding the closest row of $\Delta_{\rho_{c_{i}}}$ which provides lower mean square error with respect to $\Delta d$,

$$
\eta=\arg \min _{j=1, \ldots, 16}\left\|\Delta d-\left.\Delta_{\rho_{c_{i}}}\right|_{j}\right\|^{2}
$$

where $\left.\Delta_{\rho_{c_{i}}}\right|_{j}$ denotes the j-th row of the matrix $\Delta_{\rho_{c_{i}}}$. From the estimated pulse number $\eta$ it is estimated the frame number $v \in\{1, \ldots, 31\}$ which the first detected pulse belongs to. That is,

$$
v=d(\eta)-\hat{d}(1)+1
$$

Then the TOA coarse estimation can be directly identified as,

$$
\widehat{\tau}_{0}^{c}=(31-v+1) T_{f}
$$

The temporal resolution of this estimation is a frame period $T_{f}$.

\subsection{Fine TOA Estimation}

Once the beginning of the symbol is coarsely estimated, working in the frequency domain high resolution spectral estimation techniques can be applied to obtain a signal power profile, defined as the signal energy distribution with respect to the propagation delays, from which the TOA is estimated.

The fine estimation $\hat{\tau}_{0}$ is obtained from the TOA coarse estimation $\hat{\tau}_{0}^{c}$ and a high resolution time delay $\tilde{\tau}$ estimate of the first arriving path with respect to the time reference obtained in the coarse estimation stage.

$$
\hat{\tau}_{0}=\hat{\tau}_{0}^{c}+\tilde{\tau}
$$

The fine estimator consist of finding the first delay, $\tilde{\tau}$, that exceeds a given threshold, $P_{t h}$, in the power profile,

$$
\tilde{\tau}=\min \arg _{\tau}\left\{P(\tau)>P_{t h}\right\}
$$

Given the signal frequency domain structure obtained in (6), the power delay profile can be obtained by estimating the energy of the frequency domain signal filtered by the delay signature vector, $\mathbf{e}_{\tau}$, at each time delay resulting in the quadratic form,

$$
P(\tau)=\mathbf{e}_{\tau}^{H} \mathbf{R e}_{\tau}
$$

where $\mathbf{R}$ is the frequency domain signal correlation matrix. 
The quadratic form (17) allows for a low complexity implementation by applying the Fast Fourier Transform (FFT) to the following coefficients,

$$
\tilde{R}_{n}=\left\{\begin{array}{l}
\sum_{k=n+1}^{N} \mathbf{R}_{k-n, k}: 0 \leq n \leq N-1 \\
\sum_{k=1}^{N+n} \mathbf{R}_{k-n, k}:-N+1 \leq n \leq 0
\end{array}\right\}
$$

where $\tilde{R}_{n}$ is the sum of the n-th diagonal elements of the correlation matrix $\mathbf{R}$. So, the maximization problem resorts to maximize the following expression,

$$
P(\tau)=\sum_{n=1-N}^{-N+1} \tilde{R}_{n} e^{-j w_{0} \tau n}
$$

The number of points used in the DFT is equivalent to the number of values of $\tau$ to sweep. Therefore, the more points used more accurate will the TOA estimation be.

In order to obtain a more robust estimation of the correlation matrix $\mathbf{R}$, it can be obtained averaging over $K_{s}$ symbols. The expression is,

$$
\mathbf{R}=\frac{1}{N_{f} K_{s}} \sum_{k=1}^{K_{s}} \sum_{j=1}^{N_{f}} \mathbf{Y}_{j q}^{k} \mathbf{Y}_{j q}^{k}{ }^{H}
$$

Note that the computation of the correlation matrix also takes advantage of the inherent temporal diversity of the IR-UWB signal, with $N_{f}$ repeated transmitted pulses for each information symbol, by computing the correlation matrix over the $N_{f}$ received frames.

\section{Time Domain TOA Estimation}

The time domain TOA Estimator is based on the receiver's block diagram sketched in Fig. 3.

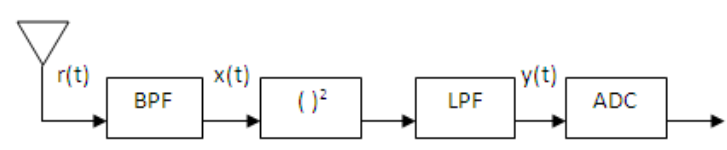

Fig. 3. Block diagram of the non-coherent receiver

The incoming waveform $r(t)$ is first passed through a BPF with center frequency $f_{0}$ and then is demodulated in a square-law device followed by a low-pass filter (LPF). The proposed ranging algorithm is based on the observation of the LPF output, which is conveniently expressed by introducing the notion of the complex envelope of a bandpass signal. 
Call $g_{l}(t)$ the convolution of $p_{l}(t)$ with the BPF impulse response, where $p_{l}(t)=h_{l} p(t)$. Now define

$$
u(t)=\sum_{l=0}^{L-1} g_{l}\left(t-\left(\tau_{l}-\tau_{0}\right)\right)
$$

Next assume that: (a) LPF has a rectangular transfer function with a bandwidth sufficiently large to pass $|\breve{x}(t)|^{2}$ undistorted; (b) the duration of $u \breve{u(t)}$ is shorter than $T_{f}$. Putting these facts together, after some manipulations it is found that the LPF output $u(t)$ can be written as the sum of a signal component $s_{y}(t)$ plus a noise component $n_{y}(t)$, with

$$
s_{y}(t)=\sum_{k=0}^{N_{S H R}-1} \sum_{j=0}^{K_{p b s}-1} c_{j}^{2} q\left(t-T_{k}^{j}-\tau_{0}\right)
$$

and $q(t)=|\breve{u}(t)|^{2}$. Note that $q(t)$ is non-negative pulse with support $\left[0, T_{f}\right)$ as a consequence of (b). Its shape is unknown and depends on the channel response.

As was done with the frequency domain estimator we assume that presence of a frame has been signaled at some time $t_{0}$ and that the estimator disposes of $K_{s}$ symbols. The TOA estimation is divided in two steps. In the first one (highestpeak search) we estimate the delay $\tau_{h}$ (relative to $t_{0}$ ) of the highest peak in the first $q(t)$-pulse of the generic symbol $N$. As is shown in Fig. 3, $\tau_{h}$ exceeds $\tau$ by some quantity $\Delta$, i.e., $\tau_{h}=\tau+\Delta$. In the second step (leading-path search) we start from the highest peak already found and, as in [5], we jump back by $T_{\text {back }}$ seconds to an instant prior to the beginning of the $q(t)$-pulse. Then, we perform a forward search looking for the first appearance of the $q(t)$-pulse energy. This provides $\Delta$, and $\tau$ is computed as $\tau=\tau_{h}-\Delta$.

\subsection{Highest-Peak Search}

To begin the Highest-Peak Search we write $\tau_{h}$ as $\tau_{h}=m T_{f}+\varepsilon$, with $0 \leq m \leq$ $K_{p b s}-1$ and $0 \leq \varepsilon<T_{f}$, and we look for the values of $m$ and $\varepsilon$. To this end, indicating with $\tilde{m}$ and $\tilde{\varepsilon}$ trial values of these parameters, we concentrate on the following function

$$
S(\tilde{m}, \tilde{\varepsilon}) \triangleq \frac{1}{K_{s}} \sum_{i=0}^{K_{s}-1} \sum_{k=0}^{K_{p b s}-1} d_{|k-\tilde{m}|_{K_{p b s}}}^{2} s_{y}\left(t_{0}+\tilde{\varepsilon}+T_{k}^{j}\right)
$$

where $|a|_{b} \triangleq a$ modulo $b$. As is now explained, its expectation $E\{S(\tilde{m}, \tilde{\varepsilon})\}$ over the thermal noise allows one to compute $m$ and $\varepsilon$. In fact, long calculations (omitted for space limitations) yield

$$
E\{S(\tilde{m}, \tilde{\varepsilon})\}=q\left(|\Delta+\tilde{\varepsilon}-\varepsilon|_{T_{f}}\right) \sum_{k=0}^{K_{p b s}-1} d_{|k-\tilde{m}|_{K_{p b s}}}^{2} d_{|k-\mu(\tilde{\varepsilon})|_{K_{p b s}}}^{2}+2 \sigma^{2} K_{ \pm 1}
$$


where $\sigma^{2}$ is the noise power at the BPF output, $K_{ \pm 1}$ is the number of non-zero elements of sequence $\left\{d_{k}\right\}_{k=0}^{K_{p b s}-1}$, and $\mu(\tilde{\varepsilon})$ is an integer defined as

$$
\mu(\tilde{\varepsilon}) \triangleq m-\left\lfloor\frac{\Delta+\tilde{\varepsilon}-\varepsilon}{T_{f}}\right\rfloor
$$

where $\lfloor x\rfloor$ is the integral part of $x$. We maintain that $E\{S(\tilde{m}, \tilde{\varepsilon})\}$ achieves its absolute maximum for $\tilde{m}=m$ and $\tilde{\varepsilon}=\varepsilon$. This is seen by inspection of (24), bearing in mind that: $(i)$ since $\Delta<T_{f}$ we have $\left\lfloor\Delta / T_{f}\right\rfloor=0$ and $|\Delta|_{T_{f}}=\Delta$; (ii) as $q(t)$ has its highest peak at $t=\Delta$ (see Fig. 3), $q\left(|\Delta+\tilde{\varepsilon}-\varepsilon|_{T_{f}}\right.$ ) has its maximum at $\tilde{\varepsilon}=\varepsilon$; (iii) for $\tilde{\varepsilon}=\varepsilon$ equation (25) gives $\mu(\tilde{\varepsilon})=m$ and the summation in (24) achieves its highest value for $\tilde{m}=m$.

In conclusion, if $E\{S(\tilde{m}, \tilde{\varepsilon})\}$ were available, $m$ and $\varepsilon$ (and hence $\tau_{h}=m T_{f}+\varepsilon$ ) could be exactly computed. As this is not the case, however, we must content ourselves with estimates. To this end we first replace $S(\tilde{m}, \tilde{\varepsilon})$ with a modified version obtained by sampling $s_{y}(t)$ at a suitable rate $1 / T_{s}$ and, next, we maximize this version instead of $E\{S(\tilde{m}, \tilde{\varepsilon})\}$. In summary, we call $t_{\ell}=t_{0}+\ell T_{s}(\ell=$ $0,1,2, \ldots)$ the sampling instants and we assume $T_{s}$ a sub-multiple of $T_{f}$, say $T_{f} / T_{s}=N_{s}$. Then, letting $\tilde{\varepsilon}=\tilde{n} T_{s}$ into the right hand side of (23) yields a quantity expressed in terms of the $s_{y}(t)$-samples. The estimates $\hat{m}$ and $\hat{\varepsilon}$ are obtained by maximizing this quantity for $0 \leq \tilde{m}<K_{p b s}-1$ and $0 \leq \tilde{n}<N_{s}-1$.

\subsection{Leading-Path Search}

Having estimated the delay $\tau_{h}$ (relative to $t_{0}$ ) of the highest peak of the first $q(t)$-pulse in the symbol $N$, we can deal with the Leading-Path Search where we look for the delay $\tau$ of the starting time of the same pulse (see Fig. 3). This can be done by measuring the difference $\Delta=\tau_{h}-\tau$. To simplify the discussion we assume that the estimate $\hat{\tau}_{h}$ of $\tau_{h}$ is perfect (i.e., $\hat{m}=m$ and $\hat{\varepsilon}=\varepsilon$ ), with the understanding that the replacements $m \rightarrow \hat{m}$ and $\varepsilon \rightarrow \hat{\varepsilon}$ must be made in the final result.

To begin, consider (23) as computed for $\tilde{m}=m$

$$
S(m, \tilde{\varepsilon})=\frac{1}{K_{s}} \sum_{i=0}^{K_{s}-1} \sum_{k=0}^{K_{p b s}-1} d_{|k-m|_{K_{p b s}}^{2}}^{2} s_{y}\left(t_{0}+\tilde{\varepsilon}+T_{k}^{j}\right)
$$

Fig. 4 shows a realization of $S(m, \tilde{\varepsilon})$ in the absence of noise. The interval $0 \leq$ $\tilde{\varepsilon} \leq T_{f}$ is divided into three parts. The rightmost one contains the bulk of the $q(t)$-pulse, with the possible exception of its right tail. The middle part is a noise-only-region (NOR). The leftmost portion supports the missing tail (if any) and is called tail region.

At first sight the following backward search [5] seems adequate to measure $\Delta$. Starting from $\tilde{\varepsilon}=\varepsilon$ one goes backward by $T_{b a c k}$ to some point in NOR. Next, a rightward search is performed until $S(m, \tilde{\varepsilon})$ crosses some threshold $\lambda$, so indicating that the beginning of the $q(t)$-pulse has been reached. When this occurs, the distance to the highest peak gives the desired estimate $\hat{\Delta}$ of $\Delta$. 


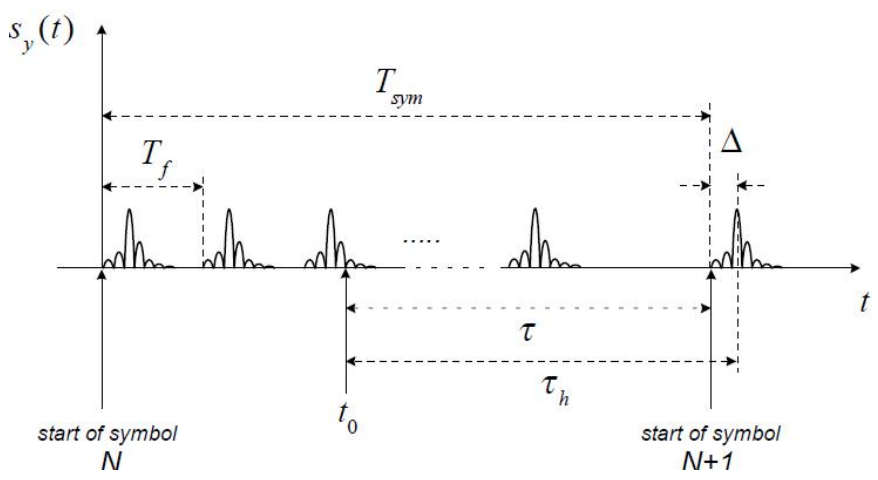

Fig. 4. Illustration of the pulses in symbol $N$.

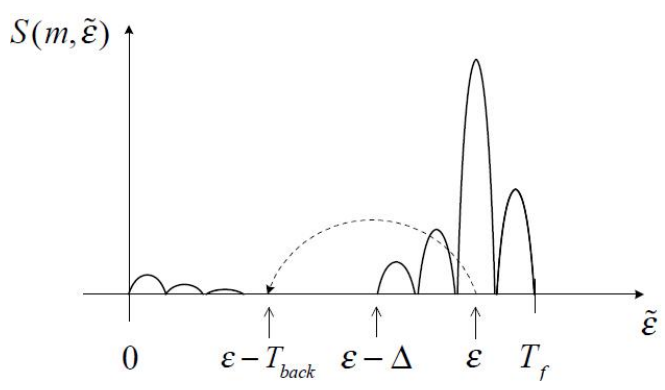

Fig. 5. Shape of $S(m, \tilde{\varepsilon})$ in the absence of noise.

Unfortunately, large errors are incurred if the search starts in the tail region rather than in the NOR. In fact, when this happens, the threshold is likely to be crossed just at the beginning of the search. An expedient to reduce the risk is to modify $S(m, \tilde{\varepsilon})$ so as to eliminate the tail region. This goal is achieved bearing in mind that the tail arises from the presence of $q(t)$-pulses at a distance $T_{f}$ in the received waveform. Thus, dropping the terms in (26) with index $k$ such that $d_{|k-m|_{K_{p b s}}}^{2}=d_{|k-m-1|_{K_{p b s}}}^{2}=1$ does the trick. As a result (26) becomes

$$
\bar{S}(m, \tilde{\varepsilon}) \triangleq \frac{1}{K_{s}} \sum_{i=0}^{K_{s}-1} \sum_{k \in \mathcal{I}(m)} d_{|k-m|_{K_{p b s}}}^{2} s_{y}\left(t_{0}+\tilde{\varepsilon}+T_{k}^{j}\right)
$$

where $\mathcal{I}(m)$ is the set of indices $k$ such that $d_{|k-m|_{K_{p b s}}}^{2}=1$ and $d_{|k-m-1|_{K_{p b s}}}^{2}=0$.

Some remarks are useful.

(a) As is done in the highest-peak search, it is convenient to proceed digitally and replace $\bar{S}(m, \tilde{\varepsilon})$ with its sampled version $\bar{S}\left(m, \tilde{n} T_{s}\right)$.

(b) A key design parameter not only here but also in the frequency domain estimator is the threshold value. If the threshold is small compared with the 
peaks, the crossing occurs before than it should and then we say that a false alarm has happened. Vice versa, if the threshold is large, the crossing occurs after than it should and the TOA is underestimated. By simulation we have found a satisfactory trade off for the value of the threshold.

\section{Numerical Results}

For numerical evaluation of the algorithm we consider the channel models developed within the framework of the IEEE 802.15.4a. In particular it is used the CM1 Residential LOS channel model. All simulations are given for 100 independent channel realizations. The main simulation parameters are shown in Table 1. The monocycle $p(t)=c(t) \cos \left(2 \pi f_{0} t\right)$ is a modulated 8th order Butterworth pulse with a $3 \mathrm{~dB}$ bandwidth of $500 \mathrm{MHz}$ and a center frequency $f_{0}=4.5$ GHz. Pulse $c(t)$ is compliant with channels 0:3, 5:6, 8:10 and 12:14 of the IEEE 802.15.4a Standard. The pulse repetition period is $T_{f}=T_{s y m} / K_{p b s}=128 \mathrm{~ns}$.

Table 1. Simulation Parameters

\begin{tabular}{|c|c|}
\hline Parameter & Value \\
\hline Pulse duration $p(t), T_{p}$ & $2 \mathrm{~ns}$ \\
Number of frames per symbol, PRF and $K_{p b s}$ & 31 \\
Preamble SYNCH length, $L_{p}$ and $N_{S Y N C}$ & 1024 symbols \\
Number of symbols in the acquisition interval, $K_{s}$ & 974 symbols \\
Symbol duration, $T_{s y m}$ & $3974.4 \mathrm{~ns}$ \\
Sampling rate, $1 / T_{s}$ & $1 \mathrm{GHz}$ \\
\hline
\end{tabular}

Fig. 6 depicts the root mean squared error (RMSE) of the estimated TOA of both estimators. Results show that an estimation accuracy of few centimeters is asymptotically achieved for high values of SNR with both estimators $(13 \mathrm{~cm}$ in frequency domain and $9.5 \mathrm{~cm}$ in time domain). Although the time domain estimator seems to be slightly more accurate than the frequency domain estimator for high SNR, it can be observed that the curve corresponding to the frequency domain estimator grows slower as the SNR decreases than the curve corresponding to the time domain estimator.

\section{Conclusion}

A comparison between time and frequency domain TOA estimators has been made. The time domain algorithm looks for the ranging marker, i.e., the arrival time of the first pulse of the physical layer header. The goal is achieved in two steps: (a) determining the arrival time of the first pulse of each symbol in the preamble; (b) locating the exact position of the start frame delimiter. The frequency domain estimator exploits the low complexity of the FFT calculation 


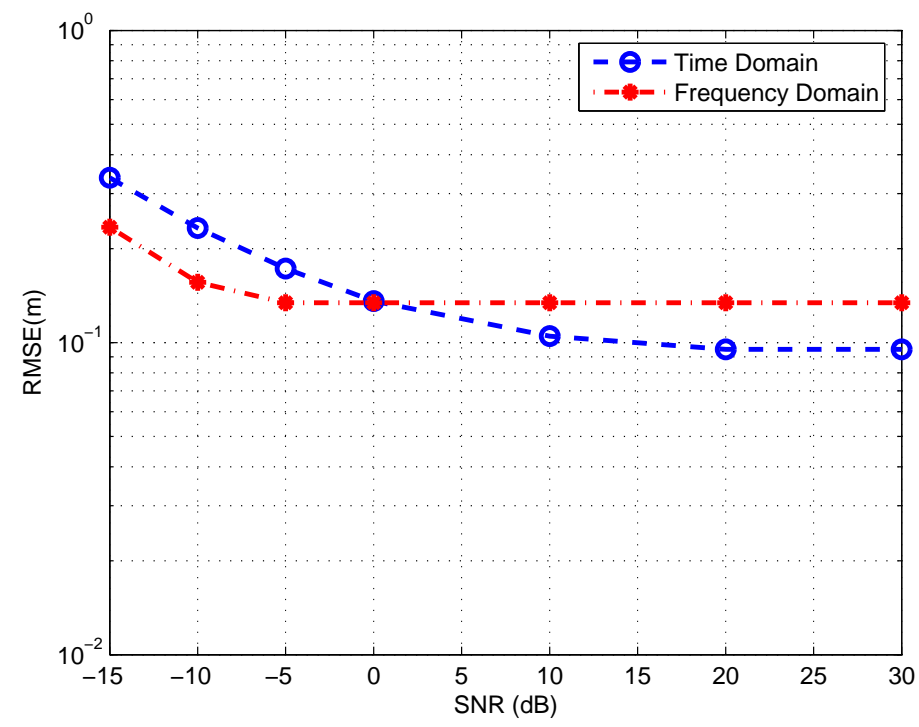

Fig. 6. RMSE $(\mathrm{m})$ of the estimated TOA of both estimators vs. SNR

by means of a power delay spectrum computation. The fine TOA estimatior requires a previous stage that provides a coarse symbols synchronization. It has been shown that time domain estimator works slightly better for high values of SNR while frequency domain estimator seems to provide more accurate results at low values of SNR.

\section{References}

1. D'Amico, A.A., Mengali, U. and Taponecco, L.: Ranging Algorithm for the IEEE 802.15.4a Standard. In Proceedings IEEE International Conference on UltraWideband, ISBN 978-1-4244-2931-8 IEEE, pp. 285-289, Vancouver, Canada, Sept. 9-11 (2009).

2. D'Amico, A.A, Mengali, U. and Taponecco, L.: TOA Estimation with the IEEE 802.15.4a Standard. Submitted to the IEEE Transactions on Wireless Communications, April 15 (2009).

3. Navarro, M. and Njar, M.: Frequency Domain Joint TOA and DOA estimation in IRUWB. Submitted for publication to the IEEE Trans. on Wireless Communications (under review), October (2008).

4. avarro, M. and Njar, M.: Joint estimation of TOA and DOA in IR-UWB. In IEEE Workshop on Signal Processing Advances in Wireless Communications, pp. 1-5, Helsinki, Finland, June 17-20 (2007).

5. D'Amico, A.A, Mengali, U. and Taponecco, L.: Energy-Based TOA Estimation. In IEEE Trans. on Wireless Communications, vol.7, no.3, pp.838-847, March (2008) 